\title{
Association between asymptomatic carriage and sporadic (endemic) meningococcal disease in an open community
}

\author{
M. E. VERDÚ ${ }^{1}$, P. COLL ${ }^{1,2 *}$, J. A. VÁZQUEZ ${ }^{3}$, F. MARCH ${ }^{1}$, D. FONTANALS ${ }^{4}$, \\ S. BERRÓN ${ }^{3}$, I. PONS ${ }^{4}$, D. VAN ESSO ${ }^{5}$ AND G. PRATS ${ }^{1,2}$ \\ ${ }^{1}$ Servei de Microbiologia, Hospital de la Santa Creu i Sant Pau, Av. Sant Antoni Maria Claret, 167, \\ 08025 Barcelona, Spain \\ ${ }^{2}$ Departament de Gẽnẽtica i Microbiologia, Universitat Autònoma de Barcelona, Barcelona, Spain \\ ${ }^{3}$ Laboratorio de Referencia de Meningococo, Centro Nacional de Microbiologia, Instituto de Salud Carlos \\ III, 28220 Majadahonda, Madrid, Spain \\ ${ }^{4}$ Laboratori de Microbiologia, Consorci Hospitalari Parc Taulí, Sabadell, Spain \\ ${ }^{5}$ Pediatria, ABS Serraparera, Cerdanyola, Spain
}

(Accepted 18 May 2001)

\section{SUMMARY}

We analysed a strain collection representative of the overall Neisseria meningitidis population circulating in an open community (46000 inhabitants, Spain) during an endemic period (30 isolates from patients and 191 from throat cultures of healthy individuals) by both phenotypic and molecular techniques. Almost all patient isolates were assigned to three hyper-virulent lineages (ET-5 complex, ET-37 complex and cluster A4) by both multilocus enzyme electrophoresis (MEE) and pulsed-field gel electrophoresis (PFGE). In contrast, MEE and PFGE assigned $20 \%$ and $15 \%$ respectively of carrier isolates to the hyper-virulent clones ( $4 \%$ for both methods together). There was also a higher correlation between PFGE and phenotypes associated with virulent clones. These notable differences between the two molecular methods were further observed in more than half the carrier isolates, suggesting that the associations between these strains were distorted by recombination events. However, almost one-third of total endemic strains from symptom-free carriers and almost all patient strains belonged to clones defined by MEE and PFGE, with no known epidemiological connection. These data indicate low transmission and a weak clonal structure for $N$. meningitidis.

\section{INTRODUCTION}

Infections due to Neisseria meningitidis are still a public health concern. Meningococci colonize the upper respiratory tract of $10-15 \%$ of the human population in open communities [1,2], occasionally invading the blood stream or spinal fluid causing disease. In Europe, most cases are sporadic (or endemic) [3] and the main causal agents are strains of

* Author for correspondence: Servei de Microbiologia, Hospital de la Santa Creu i Sant Pau, Av. Sant Antoni Maria Claret, 16708025 Barcelona, Spain. serogroup B, followed by serogroup C [4, 5]. Although the prevalence of serogroup $C$ has increased in Spain, serogroup B still prevails especially in the Mediterranean area [6].

Exposure to pathogenic meningococcal strains, together with environmental and host factors are assumed to contribute to the development of invasive disease [7]. Typing of meningococcal strains by efficient markers may improve both the understanding and the control of meningococcal disease (MD). Various methods have been used to characterize meningococci, such as serotyping [8], ribotyping [9, 10], pulsed-field 
gel electrophoresis (PFGE) [11-13], multilocus enzyme electrophoresis (MEE) [14-17], multilocus sequence typing (MLST) [18, 19], random amplified polymorphism analysis (RAP-PCR) [20] and PCRrestriction fragment length polymorphism analysis $[9$, 21].

Before the development of MLST, a method based on DNA sequencing, MEE was the method of choice for analysing bacterial population structures and global epidemiology [14, 22]. Most serogroup B or C isolates associated with invasive meningococcal disease in the developed world have been assigned to a small number of hyper-virulent lineages, referred to as ET-5 complex, ET-37 complex, and cluster A4 [15, 16]. Recently, these major lineages have also been identified by MLST [18]. In addition, several studies have shown that methods such as PFGE, which are very sensitive to microevolution, are useful for shortterm epidemiology [11, 23].

Although transmission of virulent clones through human populations during endemic periods has been inferred from a disease-typing study [24], the whole $N$. meningitidis population from an endemic area, i.e. strains from both healthy carriers and patients, has not been characterized. In the context of a period of hyperendemic disease in Norway, Caugant et al. [25] used MEE to compare isolates from healthy carriers in a localized community (Trømso) and patients with MD from all over the country and found a low frequency of carriage of hyper-virulent clones (ET-5 and ET-37 complexes). However, when isolates from healthy military recruits were examined, ET- 5 complex prevailed amongst both carriers and cases [26].

To characterize the overall $N$. meningitidis population accurately, both a suitable typing method and a representative strain collection have to be selected. Most studies have focused on a small number of lineages that are responsible for most cases of the disease, and so have been performed with highly biased samples, in which these lineages are overrepresented $[18,27]$. This is likely to underestimate the diversity of the population as a whole and hinder our understanding of both the dynamics of meningococcal carriage and the connection between such carriage and invasive meningococcal disease.

Here, we report an analysis of the phenotypic and genotypic composition of a representative strain collection of the overall $N$. meningitidis population recovered from a localized open community during an endemic period. Using serology, MEE and PFGE, we characterized a meningococcal strain collection obtained from all cases of MD reported between 1987 and 1993 [11] and from a cross-sectional seasonal carriage survey carried out between March 1992 and January 1993 in a Spanish town (Cerdanyola, 46000 inhabitants) [2].

\section{MATERIALS AND METHODS}

\section{Population and data collection}

Between 1987 and 1993, when no localized outbreaks were reported, 36 cases of MD were notified in Cerdanyola, Barcelona, Spain (population 46000). $N$. meningitidis was isolated from 30 of these 36 patients. Meningococci were not obtained by culture from the remaining six [11].

Throat samples were collected from 1500 individuals between March 1992 and January 1993 [2]. In order to include different age and social groups, the sampling survey was carried out in day-care centres, schools, colleges, and cultural and working centres in various areas (residential, central and peripheral) in Cerdanyola in four campaigns (March, June, September and January). N. meningitidis was identified in 191 of these throat cultures and one colony from each carrier was kept at $-80^{\circ} \mathrm{C}$. None of these individuals had had contact with a MD case. The overall meningococcal carriage rate was $10.5 \%$ [2].

\section{Serogrouping, serotyping and subtyping}

Serogrouping was performed by standard slide agglutination. Serotypes and subtypes were determined by a whole-cell ELISA. Antigens were prepared as described by Abdillahi and Poolman [28]. Monoclonal antibodies with serotype specificities $1,2 \mathrm{a}, 2 \mathrm{~b}, 2 \mathrm{c}, 4$, 14 and 15 and subtype specificities P1.1, P1.2, P1.3, P1.4, P1.6, P1.7, P1.9, P1.10, P1.12, P1.13, P.14, P1.15 and P1.16 were supplied by Dr J. T. Poolman (RIVM, Bilthoven, the Netherlands).

\section{Enzyme electrophoresis}

Enzyme extraction, polyacrylamide gel electrophoresis and selective enzyme staining were performed as described elsewhere [29]. Relative enzyme mobilities for each of the eight enzymes assayed (alleles) were numbered in order of decreasing anodal mobility and each unique set of alleles was defined as an electrophoretic type (ET). At a maximal genetic distance of 
Table 1. Characteristics of 221 isolates of $\mathrm{N}$. meningitidis from carriers and MD cases

\begin{tabular}{|c|c|c|c|c|c|c|c|c|c|c|c|c|c|}
\hline \multicolumn{2}{|c|}{ Isolate no. } & \multicolumn{8}{|c|}{ Allele at the following enzyme locus } & \multirow[b]{2}{*}{ ET } & \multirow[b]{2}{*}{ PT } & \multirow{2}{*}{$\begin{array}{l}\text { Serogroup: } \\
\text { serotype: } \\
\text { subtype }\end{array}$} & \multirow{2}{*}{$\begin{array}{l}\text { Cluster } \\
\text { no.* }\end{array}$} \\
\hline Patient & Carrier & G6P & ME & $\mathrm{ADH}$ & GD1 & GD2 & IDH & ALP & $\mathrm{MDH}$ & & & & \\
\hline & 108 & 5 & 1 & 2 & 4 & 1 & 1 & 4 & 2 & 1 & $A_{1}$ & NG:NT:P1.15 & 1 \\
\hline & 1378 & 5 & 1 & 2 & 3 & 2 & 1 & 4 & 2 & 2 & $\mathrm{AV}_{1}$ & NG:NT:NST & \\
\hline & 1457 & 5 & 1 & 2 & 3 & 2 & 1 & 4 & 2 & & $\mathrm{AV}_{2}$ & NG:NT:NST & \\
\hline & 109 & 2 & 2 & 2 & 3 & 2 & 4 & 1 & 2 & 3 & $\mathrm{AB}_{1}$ & B:4:P1.14 & 2 \\
\hline & 241 & 2 & 2 & 2 & 3 & 2 & 2 & 1 & 2 & 4 & $\mathrm{C}_{1}$ & B:1:P1.14 & \\
\hline & 95 & 2 & 2 & 2 & 3 & 2 & 3 & 1 & 2 & 5 & $A_{1}$ & NG:4:P1.14,16 & \\
\hline & 697 & 2 & 2 & 2 & 3 & 2 & 3 & 4 & 2 & 6 & $\mathrm{AX}_{3}$ & $\mathrm{~B}: 1: \mathrm{NST}$ & \\
\hline & 700 & 2 & 2 & 2 & 3 & 2 & 3 & 4 & 2 & & $\mathrm{AX}_{3}$ & B:1:NST & \\
\hline & 267 & 1 & 2 & 2 & 3 & 2 & 2 & 1 & 2 & 7 & $\mathrm{~V}_{1}^{3}$ & B:4:NST & \\
\hline & 1128 & 1 & 2 & 2 & 3 & 2 & 4 & 1 & 2 & 8 & $\mathrm{~V}_{2}$ & B:NT:P1.14 & \\
\hline & 306 & 1 & 2 & 2 & 3 & 2 & 3 & 1 & 2 & 9 & $\mathrm{C}_{1}$ & B:1:P1.14 & \\
\hline & 325 & 1 & 2 & 2 & 3 & 2 & 3 & 1 & 2 & & $\mathrm{C}_{2}$ & B:4:P1.14 & \\
\hline & 268 & 1 & 2 & 2 & 3 & 2 & 3 & 1 & 2 & & $\mathrm{C}_{4}$ & NG:4:P1.14 & \\
\hline & 490 & 1 & 2 & 2 & 3 & 2 & 5 & 4 & 2 & 10 & $\mathrm{AX}_{5}$ & B:NT:NST & \\
\hline & 444 & 2 & 1 & 2 & 2 & 2 & 1 & 4 & 2 & 11 & $\mathrm{AH}_{1}$ & B:4:P1.4 & 3 \\
\hline & 486 & 2 & 1 & 2 & 2 & 2 & 1 & 4 & 2 & & $\mathrm{AH}_{1}$ & B:4:P1.4 & \\
\hline & 528 & 2 & 1 & 2 & 2 & 2 & 1 & 4 & 2 & & $\mathrm{AH}_{1}$ & B:4:P:1.1,2 & \\
\hline & 470 & 2 & 1 & 2 & 2 & 2 & 1 & 4 & 2 & & $\mathrm{AH}_{2}$ & B:4:P1.1,2 & \\
\hline & 473 & 2 & 1 & 2 & 2 & 2 & 1 & 4 & 2 & & $\mathrm{AH}_{2}$ & B:4:P1.1,2 & \\
\hline & 1340 & 2 & 1 & 2 & 2 & 2 & 3 & 4 & 2 & 12 & $\mathrm{AH}_{4}$ & NG:4:P1.2 & \\
\hline & 594 & 2 & 1 & 2 & 3 & 2 & 4 & 4 & 2 & 13 & $\mathrm{H}$ & B:4:P1.6 & \\
\hline & 508 & 2 & 2 & 2 & 2 & 2 & 2 & 4 & 2 & 14 & $\mathrm{AH}_{1}$ & B:4:P1.1,2 & \\
\hline & 162 & 2 & 2 & 2 & 2 & 2 & 1 & 1 & 2 & 15 & $P_{1}$ & B:NT:P1.16 & 4 \\
\hline & 1233 & 3 & 2 & 2 & 2 & 2 & 1 & 1 & 2 & 16 & $\mathrm{~W}_{1}$ & $\mathrm{~B}: 14: \mathrm{P} 1.2$ & \\
\hline & 808 & 5 & 2 & 2 & 2 & 2 & 3 & 1 & 2 & 17 & $\mathrm{C}_{3}$ & B:4:P1.14 & \\
\hline & 242 & 3 & 2 & 2 & 2 & 2 & 2 & 3 & 2 & 18 & $\mathrm{C}_{1}^{3}$ & NG:4:P1.9 & \\
\hline & 1220 & 3 & 2 & 2 & 2 & 2 & 2 & 2 & 2 & 19 & $\mathrm{~W}_{2}$ & B:14:NST & \\
\hline & 836 & 3 & 2 & 2 & 2 & 2 & 2 & 2 & 2 & & $\mathrm{~W}_{3}^{2}$ & B:14:NST & \\
\hline & 244 & 3 & 2 & 2 & 2 & 2 & 2 & 2 & 2 & & $\mathrm{AS}_{1}$ & NG:15:P1.6 & \\
\hline & 636 & 3 & 2 & 2 & 2 & 2 & 2 & 2 & 2 & & $\mathrm{AS}_{4}$ & B:4:P1.6 & \\
\hline & 758 & 3 & 2 & 2 & 2 & 2 & 2 & 2 & 2 & & $\mathrm{AS}_{5}$ & B:4:NST & \\
\hline & 1277 & 3 & 2 & 2 & 2 & 2 & 2 & 2 & 2 & & $\mathrm{AW}_{1}$ & B:4:NST & \\
\hline & 1358 & 3 & 2 & 2 & 2 & 2 & 2 & 2 & 2 & & $\mathrm{AW}_{1}$ & B:4:P1.6 & \\
\hline & 495 & 3 & 2 & 2 & 2 & 2 & 2 & 2 & 2 & & $\mathrm{AJ}_{1}$ & B:14:NST & \\
\hline & 1054 & 3 & 2 & 2 & 2 & 2 & 2 & 5 & 2 & 20 & $\mathrm{AF}_{7}$ & B:1:P1.6 & \\
\hline & 271 & 3 & 2 & 2 & 2 & 2 & 2 & 5 & 2 & & $\mathrm{AK}_{2}$ & B:4:P1.9 & \\
\hline & 1390 & 3 & 2 & 2 & 2 & 2 & 2 & 5 & 2 & & $\mathrm{AP}_{1}$ & B:1:NST & \\
\hline & 243 & 3 & 2 & 2 & 2 & 2 & 2 & 5 & 2 & & $\mathrm{AP}_{3}$ & NG:1:P1.6 & \\
\hline & 161 & 3 & 2 & 2 & 2 & 2 & 4 & 3 & 2 & 21 & $\mathrm{~S}_{1}$ & NG:4:P1.7,9 & \\
\hline & 464 & 3 & 2 & 2 & 2 & 2 & 4 & 3 & 2 & & $\mathrm{~S}_{2}$ & B:4:P1.9 & \\
\hline & 50 & 3 & 2 & 2 & 2 & 2 & 4 & 2 & 2 & 22 & $\mathrm{~F}_{1}$ & B:1:P1.6 & \\
\hline & 54 & 3 & 2 & 2 & 2 & 2 & 4 & 2 & 2 & & $\mathrm{~F}_{1}$ & B:1:NST & \\
\hline & 1546 & 3 & 2 & 2 & 2 & 2 & 4 & 2 & 2 & & $\mathrm{AJ}_{1}$ & NG:1:P1.10 & \\
\hline \multirow[t]{10}{*}{889638} & & 3 & 2 & 2 & 2 & 3 & 4 & 2 & 2 & 23 & $\mathrm{Y}_{1}$ & B:NT:NST & \\
\hline & 827 & 3 & 2 & 2 & 2 & 3 & 4 & 2 & 2 & & $\mathrm{AP}_{1}$ & B:1:NST & \\
\hline & 1152 & 2 & 2 & 2 & 2 & 2 & 4 & 2 & 2 & 24 & $\mathrm{AF}_{3}$ & B:NT:P1.16 & \\
\hline & 344 & 3 & 2 & 2 & 2 & 2 & 3 & 4 & 2 & 25 & $\mathrm{~N}_{1}$ & $\mathrm{C}: 2 \mathrm{~b}: \mathrm{NST}$ & \\
\hline & 40 & 3 & 2 & 2 & 2 & 2 & 3 & 2 & 2 & 26 & $\mathrm{AC}$ & B:1:NST & \\
\hline & 990 & 3 & 2 & 2 & 2 & 2 & 3 & 2 & 2 & & $\mathrm{AP}_{1}$ & $\mathrm{~B}: 1: \mathrm{NST}$ & \\
\hline & 655 & 4 & 2 & 2 & 2 & 2 & 3 & 2 & 2 & 27 & $\mathrm{I}_{5}$ & C:NT:P1.6 & \\
\hline & 840 & 4 & 2 & 2 & 2 & 2 & 3 & 2 & 2 & & $\mathrm{~T}$ & NG.NT.P1.6 & \\
\hline & 568 & 5 & 1 & 2 & 2 & 2 & 4 & 2 & 2 & 28 & M & B:4:NST & 5 \\
\hline & 1370 & 3 & 1 & 2 & 2 & 2 & 4 & 2 & 2 & 29 & $\mathrm{AG}$ & B:1:P1.15 & \\
\hline
\end{tabular}


Table 1 (cont.)

\begin{tabular}{|c|c|c|c|c|c|c|c|c|c|c|c|c|c|}
\hline \multicolumn{2}{|c|}{ Isolate no. } & \multicolumn{8}{|c|}{ Allele at the following enzyme locus } & \multirow[b]{2}{*}{ ET } & \multirow[b]{2}{*}{ PT } & \multirow{2}{*}{$\begin{array}{l}\text { Serogroup: } \\
\text { serotype: } \\
\text { subtype }\end{array}$} & \multirow{2}{*}{$\begin{array}{l}\text { Cluster } \\
\text { no.* }\end{array}$} \\
\hline Patient & Carrier & G6P & ME & $\mathrm{ADH}$ & GD1 & GD2 & IDH & ALP & $\mathrm{MDH}$ & & & & \\
\hline & 680 & 5 & 3 & 2 & 2 & 2 & 3 & 2 & 2 & 30 & $\mathrm{AY}_{1}$ & $\mathrm{~B}: \mathrm{NT}: \mathrm{NST}$ & \\
\hline & 204 & 2 & 2 & 2 & 3 & 2 & 2 & 2 & 2 & 31 & $\mathrm{AT}_{2}$ & B:1:P1.6 & 6 \\
\hline & 536 & 3 & 2 & 2 & 3 & 2 & 2 & 2 & 2 & 32 & $\mathrm{AS}_{6}^{2}$ & B:4:P1.6 & \\
\hline & 319 & 3 & 2 & 2 & 3 & 2 & 2 & 2 & 2 & & $\mathrm{AT}_{1}$ & B:NT:P1.6 & \\
\hline & 721 & 3 & 2 & 2 & 3 & 2 & 2 & 2 & 2 & & $\mathrm{AU}_{2}$ & B:4:P1.6 & \\
\hline & 1294 & 3 & 2 & 2 & 3 & 2 & 2 & 2 & 2 & & $\mathrm{E}$ & B:NT:NST & \\
\hline & 356 & 3 & 1 & 2 & 3 & 2 & 2 & 2 & 2 & 33 & $\mathrm{AF}_{2}$ & C:NT:P1.1,2 & \\
\hline & 388 & 3 & 2 & 2 & 3 & 2 & 1 & 2 & 2 & 34 & $\mathrm{AU}_{3}^{2}$ & B:4:P1.6 & \\
\hline & 326 & 3 & 2 & 2 & 3 & 2 & 3 & 2 & 2 & 35 & $\mathrm{AK}_{5}^{\circ}$ & B:4:P1.6 & \\
\hline & 694 & 3 & 2 & 2 & 3 & 2 & 3 & 2 & 2 & & $\mathrm{AS}_{5}$ & $\mathrm{~B}: 4: \mathrm{NST}$ & \\
\hline & 947 & 3 & 2 & 2 & 3 & 2 & 3 & 2 & 2 & & $\mathrm{AT}_{1}$ & $\mathrm{~B}: 4: \mathrm{P} 1.6$ & \\
\hline & 565 & 3 & 2 & 2 & 3 & 2 & 3 & 2 & 2 & & $\mathrm{AV}_{1}$ & B:4:P1.6 & \\
\hline & 843 & 3 & 2 & 2 & 3 & 2 & 3 & 2 & 2 & & $\mathrm{AW}_{2}$ & B:4:P1.6 & \\
\hline & 276 & 4 & 2 & 2 & 3 & 2 & 4 & 2 & 2 & 36 & $\mathrm{R}_{1}$ & NG:NT:NST & \\
\hline & 1164 & 4 & 1 & 2 & 3 & 2 & 4 & 2 & 2 & 37 & $\mathrm{AF}_{1}$ & B:4:P1.6 & \\
\hline & 266 & 3 & 2 & 2 & 3 & 2 & 5 & 2 & 2 & 38 & $\mathrm{AK}_{6}$ & NG:15:P1.6 & 7 \\
\hline & 348 & 3 & 2 & 2 & 3 & 2 & 5 & 2 & 2 & & $\mathrm{AL}_{1}$ & B:NT:NST & \\
\hline & 28 & 3 & 2 & 2 & 3 & 2 & 5 & 1 & 2 & 39 & $\mathrm{~K}^{1}$ & B:4:NST & \\
\hline & 36 & 3 & 2 & 2 & 4 & 2 & 5 & 1 & 2 & 40 & $\mathrm{~K}$ & $\mathrm{~B}: 4: \mathrm{NST}$ & \\
\hline & 462 & 3 & 2 & 2 & 4 & 2 & 2 & 4 & 2 & 41 & $\mathrm{~N}_{1}$ & NG:2a:NST & \\
\hline & 639 & 3 & 2 & 2 & 4 & 2 & 2 & 4 & 2 & & $\mathrm{~N}_{1}$ & $\mathrm{C}: 2 \mathrm{~b}: \mathrm{NST}$ & \\
\hline & 454 & 3 & 2 & 2 & 4 & 2 & 2 & 4 & 2 & & $\mathrm{~N}_{3}$ & NG:2a:NST & \\
\hline & 250 & 3 & 2 & 2 & 4 & 2 & 2 & 4 & 2 & & $\mathrm{~N}_{4}$ & $\mathrm{C}: 2 \mathrm{~b}: \mathrm{NST}$ & \\
\hline & 1613 & 3 & 2 & 2 & 3 & 2 & 2 & 4 & 2 & 42 & $\mathrm{~N}_{1}^{4}$ & NG:2b:NST & \\
\hline & 185 & 3 & 2 & 2 & 3 & 2 & 2 & 4 & 2 & & $\mathrm{~N}_{1}$ & NG:2b:NST & \\
\hline & 654 & 3 & 2 & 2 & 3 & 2 & 2 & 4 & 2 & & $\mathrm{~N}_{2}$ & $\mathrm{C}: 2 \mathrm{~b}: \mathrm{NST}$ & \\
\hline & 1290 & 3 & 2 & 2 & 3 & 2 & 2 & 4 & 2 & & $\mathrm{AO}_{1}$ & $\mathrm{~B}: 2 \mathrm{~b}: \mathrm{P} 1.10$ & \\
\hline \multirow[t]{2}{*}{$9304432 \dagger$} & & 3 & 2 & 2 & 3 & 2 & 4 & 4 & 2 & 43 & $\mathbf{A O}_{1}$ & B:2b:P1.10 & \\
\hline & 498 & 3 & 2 & 2 & 3 & 2 & 4 & 4 & 2 & & $\mathrm{AO}_{2}$ & $\mathrm{C}: 2 \mathrm{~b}: \mathrm{P} 1.1,2$ & \\
\hline \multirow[t]{23}{*}{9315144} & & 3 & 2 & 2 & 3 & 2 & 4 & 4 & 2 & & $\mathrm{AO}_{3}$ & C:2b:P1.2 & \\
\hline & 59 & 3 & 2 & 2 & 4 & 2 & 3 & 4 & 2 & 44 & $\mathrm{~N}_{1}$ & $\mathrm{C}: 2 \mathrm{~b}: \mathrm{NST}$ & \\
\hline & 665 & 3 & 1 & 2 & 1 & 2 & 3 & 2 & 2 & 45 & $\mathrm{I}_{4}$ & Y:NT:P1.6 & 8 \\
\hline & 132 & 3 & 2 & 2 & 1 & 2 & 3 & 2 & 2 & 46 & $\mathrm{I}_{1}$ & NG:NT:NST & \\
\hline & 262 & 3 & 2 & 2 & 1 & 2 & 3 & 2 & 2 & & $\mathrm{I}_{1}$ & B:1:P1.6 & \\
\hline & 1392 & 3 & 2 & 2 & 1 & 2 & 2 & 2 & 2 & 47 & $\mathrm{I}_{2}$ & NG:NT:NST & \\
\hline & 434 & 3 & 2 & 2 & 1 & 2 & 2 & 2 & 2 & & $\mathrm{I}_{2}$ & NG:NT:P1.6 & \\
\hline & 659 & 3 & 2 & 2 & 1 & 2 & 2 & 2 & 2 & & $\mathrm{AY}_{1}$ & NG:NT:P1.6 & \\
\hline & 667 & 3 & 2 & 2 & 1 & 2 & 4 & 2 & 2 & 48 & $\mathrm{I}_{2}$ & NG:NT:P1.6 & \\
\hline & 1478 & 3 & 2 & 2 & 1 & 2 & 4 & 2 & 2 & & $\mathrm{~J}$ & $\mathrm{~B}: 4: \mathrm{P} 1.4$ & \\
\hline & 1053 & 3 & 2 & 2 & 1 & 2 & 1 & 1 & 2 & 49 & $\mathrm{~L}_{1}$ & B:NT:P1.6 & \\
\hline & 1346 & 3 & 2 & 2 & 1 & 2 & 2 & 1 & 2 & 50 & $\mathrm{I}_{1}$ & NG:4:P1.6 & \\
\hline & 658 & 2 & 1 & 2 & 1 & 2 & 5 & 2 & 2 & 51 & $\mathrm{Q}_{1}$ & NG:4:P1.16 & - \\
\hline & 118 & 5 & 2 & 2 & 2 & 3 & 3 & 2 & 2 & 52 & $\mathrm{U}_{1}$ & NG:4:NST & 9 \\
\hline & 871 & 4 & 2 & 2 & 2 & 3 & 3 & 2 & 2 & 53 & $\mathrm{U}_{1}$ & NG:4:NST & \\
\hline & 638 & 4 & 2 & 2 & 2 & 3 & 3 & 2 & 2 & & $Y_{2}$ & Y:14:NST & \\
\hline & 482 & 4 & 2 & 2 & 3 & 3 & 3 & 2 & 2 & 54 & $\mathrm{U}_{1}^{2}$ & $\mathrm{~B}: 4: \mathrm{NST}$ & \\
\hline & 810 & 3 & 3 & 2 & 2 & 3 & 2 & 2 & 2 & 55 & $\mathrm{AY}_{2}$ & B:NT:NST & - \\
\hline & 110 & 5 & 1 & 4 & 2 & 3 & 4 & 1 & 4 & 56 & $A_{1}$ & $\mathrm{~B}: 4: \mathrm{P} 1.15$ & 10 \\
\hline & 99 & 3 & 1 & 4 & 2 & 3 & 4 & 1 & 4 & 57 & $\mathrm{~A}_{1}$ & NG:4:P1.15 & \\
\hline & 92 & 4 & 1 & 4 & 3 & 3 & 3 & 5 & 4 & 58 & $\mathrm{~A}_{1}$ & NG:NT:P1.15 & - \\
\hline & 390 & 2 & 1 & 2 & 2 & 3 & 4 & 1 & 2 & 59 & $\mathrm{~A}_{5}$ & B:4:P1.15 & 11 \\
\hline & 119 & 2 & 1 & 2 & 2 & 3 & 4 & 1 & 2 & & $\mathrm{D}$ & $\mathrm{B}: 4: \mathrm{P} 1.1,7$ & \\
\hline
\end{tabular}


Table 1 (cont.)

\begin{tabular}{|c|c|c|c|c|c|c|c|c|c|c|c|c|c|}
\hline \multicolumn{2}{|c|}{ Isolate no. } & \multicolumn{8}{|c|}{ Allele at the following enzyme locus } & \multirow[b]{2}{*}{ ET } & \multirow[b]{2}{*}{ PT } & \multirow{2}{*}{$\begin{array}{l}\text { Serogroup: } \\
\text { serotype: } \\
\text { subtype }\end{array}$} & \multirow{2}{*}{$\begin{array}{l}\text { Cluster } \\
\text { no.* }\end{array}$} \\
\hline Patient & Carrier & G6P & $\mathrm{ME}$ & $\mathrm{ADH}$ & GD1 & GD2 & $\mathrm{IDH}$ & ALP & $\mathrm{MDH}$ & & & & \\
\hline & 642 & 1 & 1 & 2 & 2 & 3 & 4 & 1 & 2 & 60 & $\mathrm{~A}_{6}$ & B:4:P1.15 & \\
\hline & 1375 & 1 & 1 & 2 & 2 & 3 & 4 & 1 & 2 & & $A_{8}^{6}$ & $\mathrm{~B}: 15: \mathrm{P} 1.7,16$ & \\
\hline & 263 & 1 & 1 & 2 & 3 & 3 & 4 & 1 & 2 & 61 & $\mathrm{~A}_{5}$ & NG:4:P1.15 & \\
\hline & 293 & 1 & 1 & 2 & 2 & 3 & 4 & 1 & 1 & 62 & $\mathrm{~A}_{14}$ & $\mathrm{~B}: 15: \mathrm{P} 1.7,16$ & \\
\hline & 452 & 6 & 1 & 2 & 2 & 3 & 4 & 5 & 2 & 63 & $\mathrm{~A}_{8}$ & $\mathrm{~B}: 15: \mathrm{P} 1.7,16$ & - \\
\hline & 240 & 5 & 1 & 3 & 3 & 3 & 4 & 3 & 3 & 64 & $\mathrm{O}_{1}$ & NG:15:P1.6 & 12 \\
\hline & 690 & 5 & 4 & 3 & 3 & 3 & 4 & 3 & 3 & 65 & $\mathrm{O}_{1}$ & B:15:P1.6 & \\
\hline & 720 & 3 & 1 & 3 & 3 & 3 & 1 & 3 & 3 & 66 & $\mathrm{X}_{1}$ & B:15:P1.6 & \\
\hline & 1005 & 3 & 1 & 3 & 3 & 3 & 1 & 3 & 3 & & $\mathrm{X}_{2}$ & B:15:P1.6 & \\
\hline & 1531 & 5 & 1 & 3 & 2 & 3 & 5 & 3 & 3 & 67 & $\mathrm{O}_{1}$ & B:15:P1.6 & 13 \\
\hline & 1218 & 5 & 1 & 3 & 2 & 3 & 5 & 3 & 3 & & $\mathrm{O}_{2}$ & B:15:P1.6 & \\
\hline & 296 & 5 & 4 & 3 & 2 & 3 & 5 & 3 & 3 & 68 & $\mathrm{P}_{2}^{2}$ & B:15:P1.6 & \\
\hline & 236 & 2 & 2 & 4 & 2 & 2 & 4 & 2 & 4 & 69 & $\mathrm{AK}_{1}$ & $\mathrm{~B}: 15: \mathrm{P} 1.15$ & - \\
\hline & 578 & 5 & 3 & 1 & 2 & 2 & 3 & 2 & 1 & 70 & $\mathrm{AY}_{2}$ & NG:NT:NST & 14 \\
\hline & 753 & 5 & 3 & 1 & 2 & 2 & 3 & 2 & 1 & & $\mathrm{AY}_{2}^{2}$ & NG:NT:NST & \\
\hline & 1436 & 5 & 3 & 1 & 2 & 2 & 3 & 2 & 1 & & $\mathrm{AY}{ }_{1}$ & B:4:NST & \\
\hline & 1518 & 5 & 3 & 1 & 2 & 2 & 2 & 2 & 1 & 71 & $\mathrm{AY}_{2}$ & B:4:P1.4 & \\
\hline & 1363 & 5 & 3 & 1 & 1 & 2 & 2 & 2 & 1 & 72 & $\mathrm{AY}_{1}$ & B:4:NST & \\
\hline & 509 & 5 & 3 & 3 & 2 & 2 & 2 & 2 & 3 & 73 & $\mathrm{AY}_{1}$ & NG:NT:P1.15 & - \\
\hline & 717 & 5 & 3 & 3 & 2 & 2 & 2 & 2 & 3 & & $\mathrm{AY}_{1}$ & NG:NT:NST & - \\
\hline & 496 & 4 & 2 & 3 & 1 & 2 & 2 & 2 & 3 & 74 & $\mathrm{~F}_{2}$ & B:4:P1.6 & - \\
\hline & 1522 & 3 & 2 & 3 & 3 & 2 & 5 & 2 & 3 & 75 & $\mathrm{~L}_{2}$ & $\mathrm{~B}: 4: \mathrm{P} 1.1,7$ & - \\
\hline & 1210 & 1 & 2 & 3 & 2 & 2 & 3 & 1 & 3 & 76 & $\mathrm{~V}_{1}$ & B:4:P1.14 & - \\
\hline & 1572 & 4 & 2 & 3 & 2 & 2 & 4 & 3 & 3 & 77 & $\mathrm{I}_{8}$ & $\mathrm{~B}: \mathrm{NT}: \mathrm{NST}$ & - \\
\hline & 443 & 6 & 2 & 3 & 2 & 2 & 2 & 4 & 3 & 78 & $\mathrm{AE}$ & B:1:NST & - \\
\hline & 290 & 3 & 2 & 1 & 3 & 3 & 4 & 2 & 1 & 79 & $\mathrm{U}_{2}$ & B:4:NST & 15 \\
\hline & 1560 & 3 & 2 & 1 & 3 & 2 & 4 & 2 & 1 & 80 & $\mathrm{I}_{2}{ }^{2}$ & NG:NT:P1.6 & \\
\hline & 1356 & 3 & 2 & 1 & 2 & 2 & 4 & 3 & 1 & 81 & $\mathrm{~S}_{1}$ & B:4:P1.9 & \\
\hline & 1398 & 3 & 2 & 1 & 2 & 2 & 4 & 2 & 1 & 82 & $\mathrm{AW}_{3}$ & B:4:P1.6 & \\
\hline & 79 & 3 & 2 & 1 & 2 & 2 & 4 & 2 & 1 & & $\mathrm{AP}_{1}{ }^{\circ}$ & B:1:NST & \\
\hline & 1412 & 3 & 2 & 1 & 2 & 2 & 4 & 2 & 1 & & $\mathrm{AP}_{1}$ & $\mathrm{~B}: 1: \mathrm{P} 1.2$ & \\
\hline \multirow[t]{20}{*}{9120240} & & 3 & 2 & 1 & 2 & 2 & 4 & 2 & 1 & & $\mathrm{AP}_{1}$ & B:1:P1.6 & \\
\hline & 544 & 3 & 2 & 1 & 2 & 2 & 4 & 2 & 1 & & $\mathrm{AP}_{2}$ & B:1:P1.10 & \\
\hline & 1451 & 3 & 1 & 1 & 2 & 2 & 4 & 2 & 1 & 83 & $\mathrm{AF}_{2}$ & B:NT:P1.2 & \\
\hline & 1420 & 2 & 2 & 1 & 2 & 2 & 5 & 2 & 1 & 84 & $\mathrm{AF}_{4}^{2}$ & B:NT:P1.6 & \\
\hline & 902 & 2 & 2 & 1 & 2 & 2 & 5 & 2 & 1 & & $\mathrm{AK}_{3}^{4}$ & B:NT:P1.9 & \\
\hline & 1073 & 3 & 2 & 1 & 2 & 2 & 5 & 2 & 1 & 85 & $\mathrm{AL}_{3}$ & B:NT:P1.9 & \\
\hline & 652 & 3 & 2 & 1 & 2 & 2 & 5 & 2 & 1 & & $\mathrm{AR}_{1}^{0}$ & B:NT:NST & \\
\hline & 1547 & 3 & 2 & 1 & 2 & 2 & 5 & 2 & 1 & & $\mathrm{AS}_{2}$ & B:4:P1.15 & \\
\hline & 1468 & 3 & 2 & 1 & 2 & 2 & 5 & 3 & 1 & 86 & $\mathrm{~S}_{1}$ & B:4:P1.9 & \\
\hline & 974 & 4 & 2 & 1 & 2 & 2 & 3 & 2 & 1 & 87 & $\mathrm{AK}_{7}$ & C:NT:P1.6 & \\
\hline & 1601 & 3 & 2 & 1 & 2 & 2 & 3 & 2 & 1 & 88 & $\mathrm{AK}_{1}$ & B:15:P1.15 & \\
\hline & 65 & 3 & 2 & 1 & 1 & 2 & 3 & 2 & 1 & 89 & $\mathrm{I}_{2}$ & NG:NT:P1.6 & \\
\hline & 661 & 3 & 2 & 1 & 1 & 2 & 3 & 2 & 1 & & $\mathrm{I}_{2}$ & NG:NT:P1.6 & \\
\hline & 1049 & 3 & 2 & 1 & 1 & 2 & 3 & 2 & 1 & & $I_{6}$ & B:NT:NST & \\
\hline & 1341 & 3 & 2 & 1 & 1 & 2 & 2 & 2 & 1 & 90 & $\mathrm{I}_{2}$ & NG:4:P1.6 & \\
\hline & 1395 & 3 & 2 & 1 & 2 & 2 & 2 & 2 & 1 & 91 & $\mathrm{AW}_{1}$ & B:4:P1.6 & \\
\hline & 1593 & 3 & 2 & 1 & 2 & 2 & 1 & 2 & 1 & 92 & $\mathrm{AS}_{3}$ & B:4:P1.6 & \\
\hline & 1402 & 3 & 2 & 1 & 4 & 2 & 1 & 2 & 1 & 93 & $\mathrm{Q}_{2}$ & NG:4:P1.15 & \\
\hline & 1432 & 3 & 1 & 1 & 3 & 2 & 3 & 2 & 1 & 94 & $\mathrm{AF}_{2}$ & B:NT:P1.2 & - \\
\hline & 1433 & 3 & 1 & 1 & 3 & 2 & 3 & 2 & 1 & & $\mathrm{AF}_{2}$ & B:NT:NST & - \\
\hline
\end{tabular}


Table 1 (cont.)

\begin{tabular}{|c|c|c|c|c|c|c|c|c|c|c|c|c|c|}
\hline \multicolumn{2}{|c|}{ Isolate no. } & \multicolumn{8}{|c|}{ Allele at the following enzyme locus } & \multirow[b]{2}{*}{ ET } & \multirow[b]{2}{*}{ PT } & \multirow{2}{*}{$\begin{array}{l}\text { Serogroup: } \\
\text { serotype: } \\
\text { subtype }\end{array}$} & \multirow{2}{*}{$\begin{array}{l}\text { Cluster } \\
\text { no.* }\end{array}$} \\
\hline Patient & Carrier & G6P & ME & $\mathrm{ADH}$ & GD1 & GD2 & IDH & ALP & $\mathrm{MDH}$ & & & & \\
\hline & 647 & 3 & 3 & 1 & 2 & 3 & 5 & 2 & 1 & 95 & $\mathrm{AF}_{5}$ & $\mathrm{~B}: 4: \mathrm{P} 1.4$ & 16 \\
\hline & 979 & 3 & 3 & 1 & 3 & 2 & 5 & 2 & 1 & 96 & $\mathrm{AL}_{2}$ & $\mathrm{~B}: 4: \mathrm{P} 1.4$ & \\
\hline & 402 & 2 & 2 & 1 & 3 & 2 & 3 & 1 & 1 & 97 & $\mathrm{C}_{4}$ & $\mathrm{~B}: 4: \mathrm{P} 1.14$ & 17 \\
\hline & 552 & 2 & 2 & 1 & 3 & 2 & 3 & 2 & 1 & 98 & $\mathrm{AN}$ & NG:2a:P1.1,7 & \\
\hline & 770 & 2 & 2 & 1 & 3 & 2 & 3 & 2 & 1 & & $\mathrm{I}_{3}$ & NG:2b:NST & \\
\hline & 768 & 2 & 2 & 1 & 3 & 2 & 3 & 4 & 1 & 99 & $\stackrel{\circ}{A} X_{6}$ & NG:1:NST & \\
\hline & 491 & 2 & 2 & 1 & 3 & 2 & 1 & 2 & 1 & 100 & $\mathrm{AS}_{3}$ & B:4:P1.6 & \\
\hline & 561 & 1 & 2 & 1 & 3 & 2 & 4 & 1 & 1 & 101 & $\mathrm{C}_{5}$ & NG:NT:P1.14 & \\
\hline & 748 & 2 & 2 & 1 & 3 & 2 & 4 & 1 & 1 & 102 & $\mathrm{AY}_{2}$ & B:4:P1.14 & \\
\hline & 849 & 2 & 2 & 1 & 3 & 2 & 4 & 4 & 1 & 103 & $\mathrm{AO}_{1}$ & B:NT:P1.10 & \\
\hline & 1534 & 6 & 2 & 1 & 3 & 2 & 4 & 4 & 1 & 104 & $\mathrm{AX}_{4}$ & $\mathrm{~B}: 1: \mathrm{P} 1.13$ & \\
\hline & 645 & 1 & 2 & 1 & 3 & 2 & 5 & 4 & 1 & 105 & $\mathrm{AX}_{2}$ & $\mathrm{~B}: 1: \mathrm{NST}$ & \\
\hline & 1427 & 1 & 2 & 1 & 3 & 2 & 5 & 4 & 1 & & $\mathrm{AX}_{2}^{2}$ & $\mathrm{~B}: 1: \mathrm{NST}$ & \\
\hline & 504 & 1 & 2 & 1 & 3 & 2 & 3 & 4 & 1 & 106 & $\mathrm{AX}_{1}$ & $\mathrm{~B}: 1: \mathrm{NST}$ & \\
\hline & 656 & 3 & 2 & 1 & 3 & 2 & 3 & 4 & 1 & 107 & $\mathrm{~N}_{3}$ & $\mathrm{C}: 2 \mathrm{~b}: \mathrm{NST}$ & \\
\hline 878736 & & 3 & 2 & 1 & 3 & 2 & 2 & 4 & 1 & 108 & $\mathbf{N}_{1}^{3}$ & C:2b:NST & \\
\hline $918077 \%$ & & 3 & 2 & 1 & 3 & 2 & 2 & 4 & 1 & & $\mathrm{~N}_{1}$ & C:2b:NST & \\
\hline $9121019 \ddagger$ & & 3 & 2 & 1 & 3 & 2 & 2 & 4 & 1 & & $\mathbf{N}_{1}$ & C:2b:NST & \\
\hline \multirow[t]{6}{*}{913369} & & 3 & 2 & 1 & 3 & 2 & 2 & 4 & 1 & & $\mathrm{~N}_{2}$ & C:2b:NST & \\
\hline & 1434 & 3 & 2 & 1 & 3 & 2 & 2 & 4 & 1 & & $\mathrm{~N}_{5}$ & C:2b:NST & \\
\hline & 886 & 5 & 2 & 1 & 3 & 2 & 5 & 2 & 1 & 109 & AI & B:1:P1.6 & \\
\hline & 1325 & 5 & 2 & 1 & 3 & 2 & 5 & 2 & 1 & & AI & B:1:P1.6 & \\
\hline & 25 & 3 & 2 & 1 & 3 & 2 & 5 & 1 & 1 & 110 & $\mathrm{~K}$ & $\mathrm{~B}: 4: \mathrm{NST}$ & \\
\hline & 526 & 3 & 2 & 1 & 2 & 2 & 3 & 1 & 1 & 111 & $\mathrm{G}$ & $\mathrm{B}: 15: \mathrm{P} 1.12$ & 18 \\
\hline \multirow[t]{9}{*}{9208301} & & 6 & 2 & 1 & 2 & 2 & 2 & 1 & 1 & 112 & $\mathrm{AB}_{2}$ & B:4:P1.14 & \\
\hline & 559 & 4 & 2 & 1 & 3 & 2 & 2 & 2 & 1 & 113 & $\mathrm{AJ}_{2}$ & B:NT:P1.7 & 19 \\
\hline & 891 & 4 & 1 & 1 & 2 & 2 & 2 & 2 & 1 & 114 & $\mathrm{AM}$ & $\mathrm{B}: 4: \mathrm{P} 1.15$ & \\
\hline & 903 & 4 & 1 & 1 & 1 & 2 & 4 & 2 & 1 & 115 & $\mathrm{R}_{2}$ & B:NT:NST & 20 \\
\hline & 861 & 4 & 1 & 1 & 1 & 2 & 4 & 1 & 1 & 116 & $\mathrm{Z}^{2}$ & B:NT:NST & \\
\hline & 1076 & 4 & 2 & 1 & 1 & 2 & 5 & 2 & 1 & 117 & $\mathrm{I}_{7}$ & $\mathrm{~B}: 15: \mathrm{P} 1.14$ & \\
\hline & 1449 & 4 & 1 & 1 & 1 & 2 & 5 & 2 & 1 & 118 & $\mathrm{AR}_{2}$ & B:NT:NST & \\
\hline & 1589 & 4 & 2 & 1 & 2 & 3 & 4 & 2 & 1 & 119 & $\mathrm{U}_{1}$ & $\mathrm{Y}: 4: \mathrm{P} 1.15$ & - \\
\hline & 829 & 1 & 1 & 1 & 2 & 2 & 3 & 3 & 1 & 120 & $\mathrm{~B}_{2}$ & $\mathrm{~B}: 4: \mathrm{P} 1.2$ & 21 \\
\hline \multirow[t]{3}{*}{$891529 \S$} & & 1 & 1 & 1 & 2 & 2 & 4 & 1 & 1 & 121 & $A_{10}$ & B:4:P1.15 & \\
\hline & 1407 & 2 & 1 & 1 & 2 & 3 & 4 & 1 & 1 & 122 & $\mathrm{~A}_{2}$ & $\mathrm{~B}: 4: \mathrm{P} 1 \cdot 15$ & \\
\hline & 1409 & 2 & 1 & 1 & 2 & 3 & 4 & 1 & 1 & & $\mathrm{~A}_{2}$ & $\mathrm{~B}: 4: \mathrm{P} 1 \cdot 10$ & \\
\hline $912465 \S$ & & 6 & 1 & 1 & 2 & 3 & 4 & 1 & 1 & 123 & $\mathbf{A}_{5}$ & B:4:P1.15 & \\
\hline $914324 \S$ & & 6 & 1 & 1 & 2 & 3 & 4 & 1 & 1 & & $\mathbf{A}_{5}$ & B:4:P1.15 & \\
\hline $9205370 \S$ & & 6 & 1 & 1 & 2 & 3 & 4 & 1 & 1 & & $\mathbf{A}_{5}$ & B:4:P1.15 & \\
\hline \multirow[t]{2}{*}{$87595 \S$} & & 6 & 1 & 1 & 2 & 3 & 4 & 1 & 1 & & $\mathbf{A}_{8}$ & B:15:P1.7,16 & \\
\hline & 572 & 6 & 1 & 1 & 2 & 3 & 4 & 1 & 1 & & $\mathrm{~A}_{9}$ & $\mathrm{~B}: 4: \mathrm{P} 1 \cdot 1,2$ & \\
\hline $903943 \S$ & & 1 & 1 & 1 & 2 & 3 & 4 & 1 & 1 & 124 & $\mathbf{A}_{4}$ & B:NT:P1.1,7 & \\
\hline $881601 \S$ & & 1 & 1 & 1 & 2 & 3 & 4 & 1 & 1 & & $\mathbf{A}_{5}$ & B:4:P1.15 & \\
\hline $902342 \S$ & & 1 & 1 & 1 & 2 & 3 & 4 & 1 & 1 & & $A_{5}$ & B:4:P1.15 & \\
\hline $913128 \S$ & & 1 & 1 & 1 & 2 & 3 & 4 & 1 & 1 & & $\mathbf{A}_{7}$ & B:4:P1.15 & \\
\hline $905544 \S$ & & 1 & 1 & 1 & 2 & 3 & 4 & 1 & 1 & & $\mathbf{A}_{8}$ & B:15:P1.7,16 & \\
\hline $911643 \S$ & & 1 & 1 & 1 & 2 & 3 & 4 & 1 & 1 & & $\mathbf{A}_{8}^{\circ}$ & B:15:P1.7,16 & \\
\hline $908567 \S$ & & 1 & 1 & 1 & 2 & 3 & 4 & 1 & 1 & & $A_{10}$ & B:4:P1.15 & \\
\hline $897147 \S$ & & 1 & 1 & 1 & 2 & 3 & 4 & 1 & 1 & & $\mathbf{A}_{10}$ & B:4:P1.14 & \\
\hline $888402 \S$ & & 1 & 1 & 1 & 2 & 3 & 4 & 1 & 1 & & $A_{10}$ & B:4:P1.15 & \\
\hline $904775 \S$ & & 1 & 1 & 1 & 2 & 3 & 4 & 1 & 1 & & $A_{11}$ & B:4:P1.15 & \\
\hline $9327251 \S$ & & 1 & 1 & 1 & 2 & 3 & 4 & 1 & 1 & & $\mathbf{A}_{13}$ & B:4:P1.15 & \\
\hline $912396 \S$ & & 4 & 1 & 1 & 2 & 3 & 4 & 1 & 1 & 125 & $\mathbf{A}_{3}$ & B:4:P1.15 & \\
\hline
\end{tabular}


Table 1 (cont.)

\begin{tabular}{|c|c|c|c|c|c|c|c|c|c|c|c|c|c|}
\hline \multicolumn{2}{|c|}{ Isolate no. } & \multicolumn{8}{|c|}{ Allele at the following enzyme locus } & \multirow[b]{2}{*}{ ET } & \multirow[b]{2}{*}{ PT } & \multirow{2}{*}{$\begin{array}{l}\text { Serogroup: } \\
\text { serotype: } \\
\text { subtype }\end{array}$} & \multirow{2}{*}{$\begin{array}{l}\text { Cluster } \\
\text { no.* }\end{array}$} \\
\hline Patient & Carrier & G6P & ME & $\mathrm{ADH}$ & GD1 & GD2 & IDH & ALP & $\mathrm{MDH}$ & & & & \\
\hline $9016914 \S$ & & 1 & 1 & 1 & 2 & 3 & 4 & 1 & 1 & & AQ & B:NT:P1.1,7 & \\
\hline $90595 \S$ & & 1 & 1 & 1 & 2 & 3 & 4 & 1 & 1 & & $\mathbf{A}_{8}$ & B:15:P1.7,16 & \\
\hline \multirow[t]{4}{*}{$871760 \S$} & & 4 & 1 & 1 & 2 & 3 & 4 & 1 & 1 & \multirow{4}{*}{126} & $\mathbf{A}_{3}$ & B:4:P1.15 & \\
\hline & 1521 & 4 & 1 & 1 & 2 & 3 & 5 & 1 & 1 & & $\mathrm{~B}_{1}$ & B:4:P1.15 & \\
\hline & 1018 & 4 & 1 & 1 & 2 & 3 & 5 & 1 & 1 & & $\mathrm{AF}_{6}$ & $\mathrm{~B}: 4: \mathrm{P} 1.12$ & \\
\hline & 950 & 4 & 1 & 1 & 2 & 3 & 5 & 1 & 1 & & $\mathrm{AK}_{4}$ & $\mathrm{~B}: 4: \mathrm{P} 1.12$ & \\
\hline \multirow[t]{6}{*}{ 937833§ } & & 1 & 1 & 1 & 2 & 3 & 5 & 1 & 1 & 127 & $\mathbf{A}_{5}{ }^{4}$ & B:4:P1.15 & \\
\hline & 494 & 1 & 1 & 1 & 2 & 3 & 3 & 1 & 1 & 128 & $\mathrm{~A}_{12}$ & B:15:P1.16 & \multirow{3}{*}{22} \\
\hline & 837 & 2 & 1 & 1 & 2 & 2 & 2 & 4 & 1 & 129 & $\mathrm{AH}_{3}$ & B:NT:P1.2 & \\
\hline & 1336 & 3 & 1 & 1 & 2 & 2 & 1 & 4 & 1 & 130 & $\mathrm{AH}_{1}$ & B:4:P1.2 & \\
\hline & 863 & 2 & 1 & 5 & 3 & 2 & 2 & 4 & 5 & 131 & $\mathrm{AD}$ & $\mathrm{B}: 4: \mathrm{NST}$ & - \\
\hline & 1332 & 4 & 2 & 5 & 3 & 3 & 2 & 2 & 5 & 132 & $\mathrm{AL}_{4}$ & B:NT:P1.14 & - \\
\hline
\end{tabular}

ET, electrophoretic type; PT, PFGE type or pulsetype; NG, non-groupable; NT, non-serotypable; NST, non-subtypable. Enzyme abbreviations: G6P, glucose 6-phosphate; ME, malic enzyme; ADH, alcohol dehydrogenase; GD1, nicotinamide adenine dinucleotide (NAD) phosphate-linked glutamate dehydrogenase; GD2, NAD-linked glutamate dehydrogenase; $\mathrm{IDH}$, isocitrate dehydrogenase; ALP, alkaline phosphatase; $\mathrm{MDH}$, malate dehydrogenase

* The dash indicates an unrelated ET.

Disease-associated strains correlated with the cluster A4†, ET-37 complexł, and ET-5 complex§ [11] are indicated in bold type.

$0 \cdot 34$, all members of the ET-5 complex [11] were included (criteria for interpretation of closely related ETs).

\section{Pulsed-field gel electrophoresis}

DNA for PFGE analysis was prepared as described elsewhere [11]. The inserts were subjected to digestion with NheI (Amersham Pharmacia Biotech, Uppsala, Sweden) [11]. PFGE was performed by orthogonal field-alternation electrophoresis (Gene Navigator, Pharmacia LKB Biotechnology, Uppsala, Sweden) for $18 \mathrm{~h}$ at a constant $250 \mathrm{~V}$ and $12{ }^{\circ} \mathrm{C}$; the pulse time was 20, 10 and $2 \mathrm{sec}$ for 9,7 and $2 \mathrm{~h}$, respectively. Bacteriophage lambda concatemers (New England BioLabs Inc., Beverly, MA, USA) were used as size standards.

The analysis of PFGE data was facilitated by a computerized image analysis system (Bioimage, Ann Arbor, MI, USA). A dendrogram was constructed using the unweighted pair group with mathematical averaging (UPGMA) method [30].

PFGE results were classified as proposed by Tenover et al. [31]. Each unrelated pattern or pulsetype (PT) (differing by $\geqslant 7$ bands) was assigned a letter code (A, B, C, etc), in alphabetical order according to the position of the PTs in the dendrogram, whereas the groups of related patterns (differing by $\leqslant 6$ bands) were given a single common letter code subdivided by a number code $\left(\mathrm{D}_{1}, \mathrm{D}_{2}, \mathrm{D}_{3}\right.$, etc). Within a subdivision (e.g. $\mathrm{D}_{1}$ ) all the strains shared a given PT.

\section{RESULTS}

Serogroup/serotype/subtype, MEE and PFGE data from 191 carriers and 30 disease-associated $N$. meningitidis strains are listed in Table 1.

\section{Phenotypic characteristics of isolates}

Of the 191 strains isolated from carriers, 44 (23\%) were non-groupable (NG) and the remaining were assigned to one of the following serogroups: $\mathrm{B}(n=$ $133,69 \cdot 6 \%), \mathrm{C}(n=11,5 \cdot 8 \%)$ or $\mathrm{Y}(n=3,1 \cdot 6 \%)$. The 30 strains from patients were assigned to either serogroup B $(n=25,83 \%)$ or C $(n=5,17 \%)$.

Six serotypes were represented among $142(74 \%)$ serotypable carrier isolates; serotype 4 predominated among both serogroup B $(62 / 133,47 \%)$ and NG isolates $(13 / 44,29.5 \%)$ and serotype $2 \mathrm{~b}$ prevailed among serogroup C $(8 / 11,73 \%)$. Serotypes $4(53 \%)$ and $2 \mathrm{~b}(20 \%)$ were also the most frequent of the four serotypes represented in the patient isolates.

Sixteen subtypes were identified among 128 (67\%) subtypable carrier isolates; subtype P1.6 (48/191; 
Table 2. Phenotypes of Neisseria meningitidis strains recovered from carriers $(\mathrm{n}=191)$ and from patients $(\mathrm{n}=30)$ in Cerdañola, Spain

\begin{tabular}{|c|c|c|c|c|c|}
\hline \multirow[b]{2}{*}{ Phenotypes* } & \multicolumn{2}{|c|}{ No. of strains $(\%)$} & \multirow[b]{2}{*}{ Phenotypes* } & \multicolumn{2}{|c|}{ No. of strains $(\%)$} \\
\hline & Carriers & Patients & & Carriers & Patients \\
\hline $\mathrm{B}: 4: \mathrm{P} 1.6$ & $16(8 \cdot 3)$ & & B:NT:P1.9 & $2(1 \cdot 0)$ & \\
\hline $\mathrm{B}: 4: \mathrm{P} 1.15$ & $7(3 \cdot 6)$ & $14(46.6)$ & $\mathrm{B}: \mathrm{NT}: \mathrm{P} 1 \cdot 1,7$ & & $2(6 \cdot 6)$ \\
\hline $\mathrm{B}: 4: \mathrm{P} 1.14$ & $6(3 \cdot 1)$ & $2(6.6)$ & B:NT:P1·14 & $2(1 \cdot 0)$ & \\
\hline B:4:P1.9 & $4(2 \cdot 0)$ & & $\mathrm{B}: \mathrm{NT}: \mathrm{P} 1.7$ & $1(0 \cdot 5)$ & \\
\hline B:4:P1.4 & $5(2 \cdot 6)$ & & B:NT:NST & $12(6 \cdot 2)$ & $1(3 \cdot 3)$ \\
\hline $\mathrm{B}: 4: \mathrm{P} 1.1,7$ & $1(0 \cdot 5)$ & & $\mathrm{C}: 2 \mathrm{~b}: \mathrm{P} 1.2$ & & $1(3 \cdot 3)$ \\
\hline $\mathrm{B}: 4: \mathrm{P} 1.1,2$ & $5(2 \cdot 6)$ & & $\mathrm{C}: 2 \mathrm{~b}: \mathrm{P} 1.1,2$ & $1(0 \cdot 5)$ & \\
\hline $\mathrm{B}: 4: \mathrm{P} 1.2$ & $2(1 \cdot 0)$ & & $\mathrm{C}: 2 \mathrm{~b}: \mathrm{NST}$ & $7(3 \cdot 6)$ & $4(13 \cdot 3)$ \\
\hline $\mathrm{B}: 4: \mathrm{P} 1.12$ & $2(1 \cdot 0)$ & & $\mathrm{C}: \mathrm{NT}: \mathrm{P} 1.1,2$ & $1(0 \cdot 5)$ & \\
\hline $\mathrm{B}: 4: \mathrm{P} 1.10$ & $1(0 \cdot 5)$ & & C:NT:P1.6 & $2(1 \cdot 0)$ & \\
\hline $\mathrm{B}: 4: \mathrm{NST}$ & $13(6 \cdot 8)$ & & Y:14:P1.15 & $1(0 \cdot 5)$ & \\
\hline $\mathrm{B}: 1: \mathrm{P} 1.6$ & $6(3 \cdot 1)$ & $1(3 \cdot 3)$ & $Y: 4: P 1.6$ & $1(0 \cdot 5)$ & \\
\hline B:1.P1.14 & $2(1 \cdot 0)$ & & Y:NT:P1.6 & $1(0 \cdot 5)$ & \\
\hline $\mathrm{B}: 1: \mathrm{P} 1.10$ & $1(0 \cdot 5)$ & & NG:4:P1.14,16 & $1(0 \cdot 5)$ & \\
\hline B:1:P1.15 & $1(0 \cdot 5)$ & & NG:4:P1.15 & $3(1 \cdot 5)$ & \\
\hline $\mathrm{B}: 1: \mathrm{P} 1.2$ & $1(0 \cdot 5)$ & & NG:4:P1.6 & $2(1 \cdot 0)$ & \\
\hline B:1:P1.13 & $1(0 \cdot 5)$ & & NG:4:P1.1,6 & $1(0 \cdot 5)$ & \\
\hline $\mathrm{B}: 1: \mathrm{NST}$ & $12(6 \cdot 2)$ & & NG:4:P1.2 & $1(0 \cdot 5)$ & \\
\hline $\mathrm{B}: 14: \mathrm{P} 1.1,7$ & $1(0 \cdot 5)$ & & NG:4:P1.9 & $1(0 \cdot 5)$ & \\
\hline $\mathrm{B}: 14: \mathrm{P} 1.2$ & $1(0 \cdot 5)$ & & NG:4:P1.7,9 & $1(0 \cdot 5)$ & \\
\hline $\mathrm{B}: 14: \mathrm{P} 1.4$ & $1(0 \cdot 5)$ & & NG:4:P1.14 & $1(0 \cdot 5)$ & \\
\hline $\mathrm{B}: 14: \mathrm{NST}$ & $3(1 \cdot 5)$ & & NG:4:NST & $2(1 \cdot 0)$ & \\
\hline $\mathrm{B}: 15: \mathrm{P} 1.15$ & $2(1 \cdot 0)$ & & NG:1:P1.6 & $1(0 \cdot 5)$ & \\
\hline $\mathrm{B}: 15: \mathrm{P} 1.7,16$ & $3(1 \cdot 5)$ & $4(13 \cdot 3)$ & NG:1:P1·10 & $1(0 \cdot 5)$ & \\
\hline $\mathrm{B}: 15: \mathrm{P} 1.6$ & $6(3 \cdot 1)$ & & NG:1:NST & $1(0 \cdot 5)$ & \\
\hline B:15:P1.16 & $1(0 \cdot 5)$ & & NG:15:P1.6 & $3(1 \cdot 5)$ & \\
\hline B:15:P1.12 & $1(0 \cdot 5)$ & & NG:2a:P1.1,7 & $1(0 \cdot 5)$ & \\
\hline $\mathrm{B}: 15: \mathrm{P} 1.14$ & $1(0 \cdot 5)$ & & NG:2a:NST & $2(1 \cdot 0)$ & \\
\hline $\mathrm{B}: 2 \mathrm{~b}: \mathrm{P} 1.10$ & $1(0 \cdot 5)$ & $1(3 \cdot 3)$ & NG:2b:NST & $3(1 \cdot 5)$ & \\
\hline B:NT:P1.16 & $2(1 \cdot 0)$ & & NG:NT:P1.6 & $7(3 \cdot 6)$ & \\
\hline B:NT:P1.6 & $3(1 \cdot 5)$ & & NG:NT:P1.15 & $3(1 \cdot 5)$ & \\
\hline B:NT:P1.2 & $3(1 \cdot 5)$ & & NG:NT:P1.14 & $1(0 \cdot 5)$ & \\
\hline B:NT:P1.10 & $1(0 \cdot 5)$ & & NG:NT:NST & $8(4 \cdot 1)$ & \\
\hline
\end{tabular}

* NG, non-groupable; NT, non-serotypable; NST, non-subtypable.

$25 \%)$ was common. Seven subtypes were observed among patient isolates, with a low frequency of subtype P1.6 $(1 / 30,3 \%)$ and a high frequency of subtype P1.15 (14/30, $47 \%)$.

Among 191 carrier strains, 64 antigenic combinations or phenotypes were detected, none of which accounted for more than $9 \%$ of the isolates. In contrast, $22(73 \%)$ of 30 patient strains belonged to phenotypes B:4:P1.15 or B:15:P1.7, $16(n=14$ and 4 respectively), and C:2b:NST $(n=4)$, which were exclusively associated with the hyper-virulent lineages ET-5 complex and ET-37 complex [11]. In contrast, these pathogenic phenotypes amounted to $17(9 \%)$ carrier strains (Table 2).

\section{Multilocus genotype analysis}

All of the eight enzyme loci were polymorphic for 3 (GD2)-6 (GD6) alleles, with a mean of 4.6 alleles per locus. MEE revealed 132 ETs: 127 ETs among the 191 carrier isolates and $10 \mathrm{ETs}$ among the 30 isolates from patients. The isolate/ET ratio was higher in patients (3.0) than in carriers $(1 \cdot 5)$. Fifty percent $(5 / 10)$ and $28 \%(36 / 127)$ of ETs from patients and carriers were represented by multiple isolates, containing $83 \%$ $(25 / 30)$ and $53 \%(101 / 191)$ of all strains, respectively. Of these ETs, only five (ETs 23, 43, 82, 108 and 123) were recovered from both patients and carriers.

The genetic relationships revealed by MEE within the overall $N$. meningitidis population are shown in 

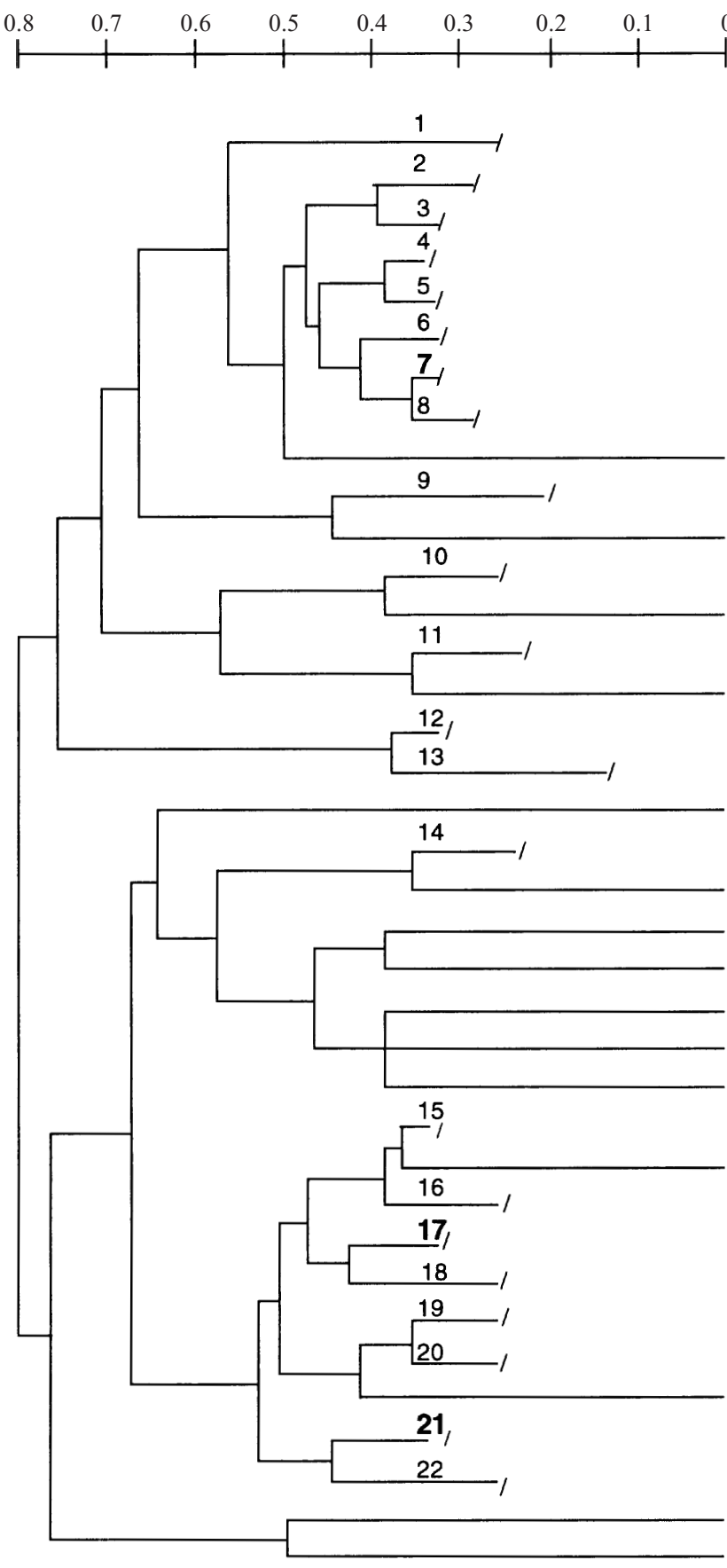

ETs

n

1-2

3

3-10

11

11-14 8

15-27

$28(+1)$

28-30 3

31-37 14

38-44 $14(+2)$

45-50 10

51

52-54 4

55

56-57 2

58

59-62

63

64-66

$67-68$

69

70-72

1
6
1
4

4

3

1

73

74

75

76

77

78

$79-93$

94

95-96

97-110

111-112

113-114

115-118

119

120-128

129-130

131

132

5

2

1

1

1

1

1

$23(+1)$

2

2

$17(+4)$

$1(+1)$

2

4

1

$8(+21)$

2

1

1

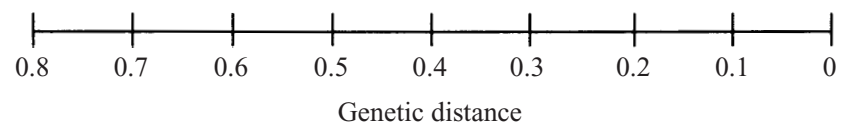

Fig. 1. Dendrogram showing genetic relationships among 132 electrophoretic types (ETs) of Neisseria meningitidis strains isolated from asymptomatic carriers and MD cases. ETs are sequentially numbered from top to bottom in the order of their listing in Table 1. Clusters represented by multiple ETs diverging at a genetic distance of $\leqslant 0 \cdot 34$ were truncated. There were 22 clusters (1-22). ET numbers and number of carrier and patient isolates (n) in each cluster are indicated (the number of patient isolates are shown in parentheses). Clones of the ET-5 complex, ET-37 complex and cluster A4 form cluster 21,17 and 7 , respectively. 
Table 3. Number (\%) of carrier and patient isolates associated with hyper-virulent lineages by multilocus enzyme electrophoresis (MEE), pulsed-field gel electrophoresis (PFGE) and by both methods together, classified by phenotypes*

\begin{tabular}{|c|c|c|c|c|c|c|c|c|c|}
\hline \multirow[b]{3}{*}{ Source } & \multicolumn{9}{|c|}{ Hyper-virulent lineages $\dagger$} \\
\hline & \multicolumn{3}{|c|}{ ET-5 complex } & \multicolumn{3}{|c|}{ ET-37 complex } & \multicolumn{3}{|c|}{ Cluster A4 } \\
\hline & MEE & PFGE & $\begin{array}{l}\text { MEE/ } \\
\text { PFGE }\end{array}$ & MEE & PFGE & $\begin{array}{l}\text { MEE/ } \\
\text { PFGE }\end{array}$ & MEE & PFGE & $\begin{array}{l}\text { MEE/ } \\
\text { PFGE }\end{array}$ \\
\hline \multicolumn{10}{|l|}{ Carriers } \\
\hline B/NG:4:P1.15 & 2 & 6 & 1 & - & - & - & - & - & - \\
\hline B:15:P1.7,16 & - & 3 & - & - & - & - & - & - & - \\
\hline C/NG:2b:NST & - & - & - & 3 & 9 & 2 & 6 & - & - \\
\hline Others & 6 & 6 & 3 & 14 & 2 & - & 8 & 3 & 2 \\
\hline Total $(n=191)$ & $8(4 \%)$ & $15(8 \%)$ & $4(2 \%)$ & $17(9 \%)$ & $11(6 \%)$ & $2(1 \%)$ & $14(7 \%)$ & $3(1 \cdot 6 \%)$ & $2(1 \%)$ \\
\hline \multicolumn{10}{|l|}{ Patients } \\
\hline B:4:P1.15 & 14 & 14 & 14 & - & - & - & - & - & - \\
\hline B:15:P1.7,16 & 4 & 4 & 4 & - & - & - & - & - & - \\
\hline $\mathrm{C}: 2 \mathrm{~b}: \mathrm{NST}$ & - & - & - & 4 & 4 & 4 & - & - & - \\
\hline Others & 3 & 2 & 2 & - & - & - & 2 & 2 & 2 \\
\hline Total $(n=30)$ & $21(70 \%)$ & $20(67 \%)$ & $20(67 \%)$ & $4(13 \%)$ & $4(13 \%)$ & $4(13 \%)$ & $2(7 \%)$ & $2(7 \%)$ & $2(7 \%)$ \\
\hline
\end{tabular}

* Phenotypes B:4:P1.15/B:15:P1.7,16 and C:2b:NST were characteristic of the ET-5 complex and ET-37 complex in our area, respectively [11]. Non-groupable (NG) carrier isolates showing these serotype:subtype combinations are included. $\dagger$ ET-5 complex corresponds to: ETs 120-128; PTs $\mathrm{A}_{1}-\mathrm{A}_{14}$. ET-37 complex corresponds to: ETs 97-110; PTs $\mathrm{N}_{1}-\mathrm{N}_{5}$. Cluster A4 corresponds to: ETs 38-44; PTs $\mathrm{AO}_{1}-\mathrm{AO}_{3}$ (see Table 1 and Figs 1 and 2).

Figure 1 . At a genetic distance $0 \cdot 34$, all patient strains and $178(93 \%)$ of 191 carrier isolates were identical or related to another. Of these 178 carrier strains, 4 were classified into 2 unrelated ETs with $>1$ isolate (ETs 73 and 94) and the remaining 174 into 22 clusters of related ETs (range 2-15 ETs, average 5 ETs), with at most $28(15 \%)$ carrier strains per cluster. Eight $(4 \%)$ carrier isolates belonged to the ET-5 complex (ETs $120-128)$, accounting for $21(70 \%)$ of 30 sporadic MD cases in the study population [11]. Of these 8, only $2(25 \%)$ were B:4:P1.15. Seventeen $(9 \%)$ and 14 (7\%) carrier isolates belonged to the ET-37 complex (ETs 97-110) and cluster A4 (ETs 38-44), which accounted for $4(13 \%)$ and $2(7 \%)$ of the disease cases, respectively. Only $3(18 \%)$ of these 17 carrier strains related to ET-37 complex were C/NG:2b:NST and 8 of the $14(57 \%)$ strains that belonged to cluster A4 were serotype $2 b$, which is characteristic of this lineage (Table 3) [16].

\section{Macrorestriction fingerprint of isolates}

When PFGE was performed using the endonuclease NheI 6-13 fragments were detected per isolate. PFGE distinguished 134 PTs; 124 PTs among the 191 carrier isolates and 16 PTs among the 30 isolates from clinical cases. The isolate/PT ratio of patients $(1.88)$ was almost as low as that of carriers (1.54). Only five PTs (PTs $\mathrm{A}_{5}, \mathrm{~A}_{8}, \mathrm{~N}_{2}, \mathrm{AO}_{1}$ and $\mathrm{AP}_{1}$ ) included both carrier and patient isolates. At a coefficient of similarity of $\geqslant 70 \%$ (i.e. $\leqslant 6$ different bands) (Fig. 2), all but 1 patient strain and $177(92.6 \%)$ of 191 carrier isolates were identical or related to another. Of these 177 carrier strains, 5 were distributed into 2 unrelated PTs with $>1$ isolate (PTs K, AI) and the remaining 172 into 33 clusters of related PTs (range: 2-14 PTs, average: $3.5 \mathrm{PTs}$ ), with at most 15 carrier strains per cluster. Specifically, these $15(8 \%)$ carrier strains were associated with those patient strains that belonged to ET-5 complex (PTs $\mathrm{A}_{1}-\mathrm{A}_{14}$ ), of which $9(73 \%)$ were either B/NG:4:P1.15 $(n=6)$ or B:15:P1.7,16 $(n=3)$. Eleven $(6 \%)$ carrier isolates were associated with those patient strains related to ET-37 complex (PTs $\left.\mathrm{N}_{1}-\mathrm{N}_{5}\right)$, of which $9(82 \%)$ were C/NG:2b:NST. Of the $3(1.6 \%)$ carrier strains associated with cluster A4 $\left(\mathrm{PTs} \mathrm{AO}_{1}-\mathrm{AO}_{3}\right), 2$ were serotype $2 \mathrm{~b}$. Only $8(4 \%)$ were assigned to the 3 pathogenic lineages by both PFGE and MEE (Table 3). 


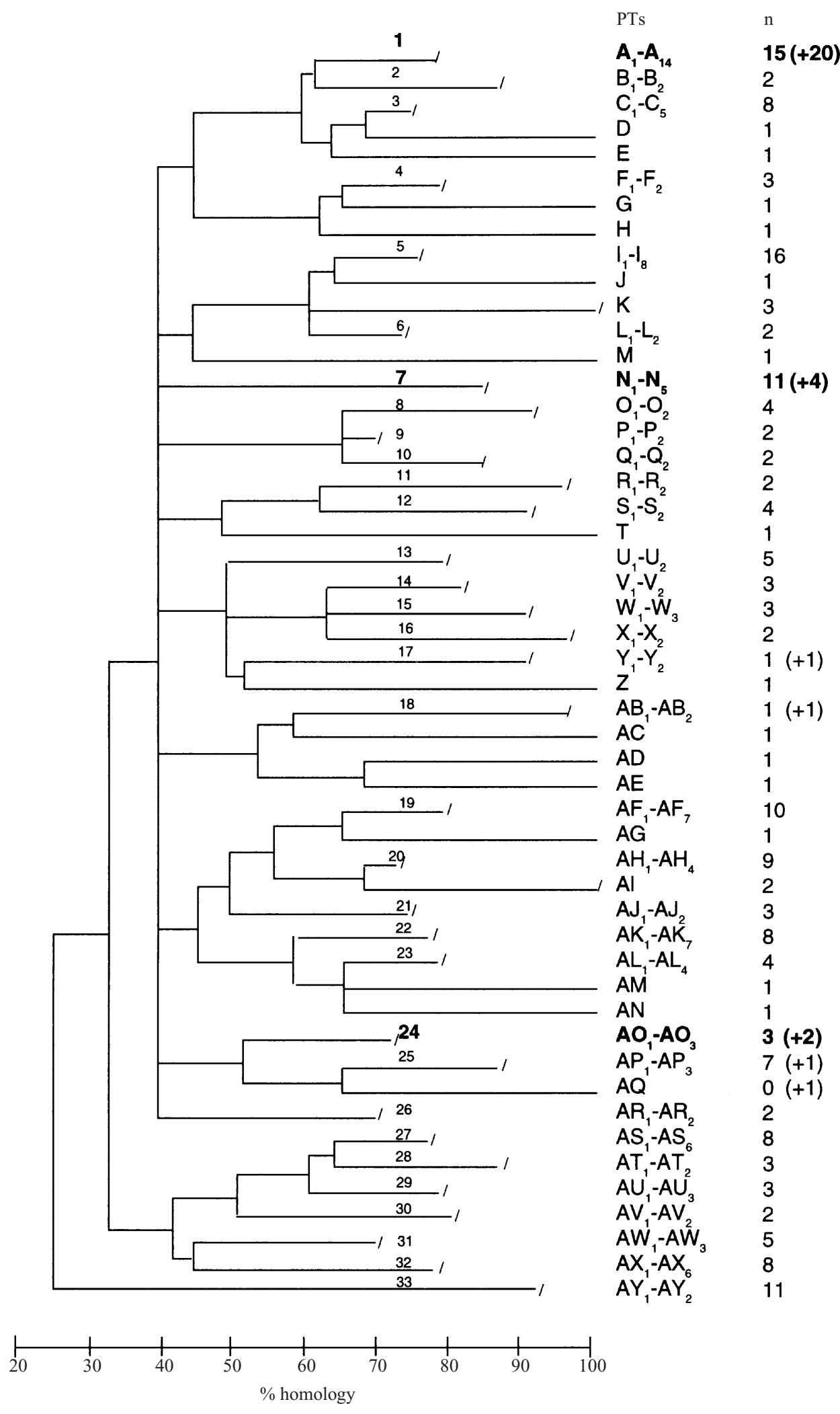

Fig. 2. Dendrogram showing the relationships between the Dice coefficients for the $221 \mathrm{~N}$. meningitidis strains as estimated by PFGE analysis. The dendrogram was generated by the UPGMA method of clustering. Numbers 1-33 indicate clusters of pulse types (PTs) that diverge at coefficient of similarity $\geqslant 70 \%$. Clones of the ET-5 complex, ET-37 complex and cluster A4 form cluster 1, 7 and 24, respectively. PT designations and numbers of carrier and patient isolates $(n)$ in each cluster are indicated (the numbers of patient isolates are shown in parentheses). 
Table 4. Electrophoretic types (ETs) represented by multiple isolates from carriers and patients associated with identical or related PFGE types*

\begin{tabular}{lllll}
\hline \hline & & & \multicolumn{2}{l}{ No. of isolates (\%) } \\
\cline { 3 - 5 } Source & ET no. $\dagger$ & No. of ETs & Total & $\begin{array}{l}\text { With identical } \\
\text { phenotype }\end{array}$ \\
\hline Carriers & $\begin{array}{l}2,6,9,11,19,20,21,22,41, \\
\end{array}$ & 23 & $57(30)$ & $27(14)$ \\
& $\begin{array}{l}82,46,47,60,66,67,70,73,94,105,109,122 \\
\text { Patients }\end{array}$ & & & \\
\hline \hline
\end{tabular}

* PFGE types within each ET, see Table 1.

$\dagger$ ET numbers are as set out in Table 1

\section{Comparison of typing systems for both carrier and sporadic meningococcal strains}

Twenty-three ETs $(23 / 36)$ recovered from more than 1 carrier and all 5 ETs causing $\geqslant 2$ sporadic cases also showed identical or related PFGE types, accounting for $57(30 \%)$ of 191 carrier strains and $24(80 \%)$ of 30 patient strains, respectively. These numbers decreased to $27(14 \%)$ and $19(63 \%)$ respectively when an identical phenotype was considered (Table 4). Of those 57 healthy carriers, an epidemiological connection (defined as isolation in the same centre) was detected in $21(37 \%)$, which harboured isolates distributed into 9 of those 23 ET type-PFGE type combinations. Conversely, 2 of these 9 combinations and the remaining 14 were identified in more than 1 centre.

The remaining $44(23 \% ; 44 / 191)$ carrier strains belonging to multiple ETs and $1(3 \% ; 1 / 30)$ patient strain, i.e. an ET-5 complex strain, had PFGE types that differed by $\geqslant 7$ bands. Conversely, 59 (31\%; 59/191) carrier isolates with distinct ET types were indistinguishable by PFGE. Of the 175 PFGE clustered carrier isolates, only $88(46 \% ; 88 / 191)$ were also related as determined by MEE (taking a genetic distance $\leqslant 0.34$ by MEE and coefficient of similarity $\geqslant 70 \%$ by PFGE as indicators of relatedness).

\section{DISCUSSION}

During the 7-year survey, when no localized outbreaks or case clusters were reported, the vast majority of isolates causing sporadic MD (70\%) belonged to the ET-5 complex by both MEE and PFGE and ET-37 complex and cluster A4 were responsible for $13 \%$ and $6.6 \%$ respectively of sporadic MD cases. Thus, most endemic cases of MD were caused by strains of a limited number of genetically defined clonal groups [11] that were also responsible for MD during hyperendemic periods [15] or epidemic outbreaks [16, 19]. These data are consistent with a gradual evolution and a slow spread of virulent meningococcal clones in geographically defined human populations over years $[24,32]$. This concept is supported by our finding of a limited number of these three hypervirulent clonal groups in the nasopharynx of symptomfree carriers (less than $20 \%$ and similar to the $16 \%$ reported by Caugant et al. [25]).

Although MEE and PFGE gave almost identical identification of virulent clones in patient isolates, concordance in carriers was less close. MEE and PFGE assigned $20 \%$ and $15 \%$ of carrier isolates to the hyper-virulent lineages respectively but only $4 \%$ were assigned by both methods. In an attempt to resolve this discrepancy, we compared phenotypes. A correlation has been established between phenotypes and pathogenic clones in limited geographical areas [32-34]. In our area, B:4:P1.15/B:15:P1.7,16 and C:2b:NST characterized the ET-5 complex and ET-37 complex, respectively. These phenotypes were more prevalent among carriers associated with these pathogenic clones by PFGE $(69 \%)$ than by MEE $(20 \%)$. Thuse, PFGE may be the more reliable marker to determine the dissemination of virulent clones in open communities.

Although MEE and PFGE give varying results on the genetic clustering of the overall $N$. meningitidis population, approximately $90 \%$ of this population was distributed into 22 and 33 clusters of related ETs and PTs, respectively. Except for one patient who had 
a unique unrelated PT, all patient strains were identical or related (by both techniques) to other isolates from carriers, who had had no known contact with MD patients. Thus, neither technique split patient and carrier isolates into two separate subpopulations. However, patient strains were phenotypically and genotypically more homogeneous than carrier strains.

Almost $75 \%$ of invasive strains belonged to the three phenotypes characteristic of the ET-5 complex and ET-37 complex in our area (as mentioned above). In contrast, these phenotypes constituted only $9 \cdot 1 \%$ of carrier strains, amongst which no phenotype had a prevalence higher than $9 \%$. This increased antigenic diversity among carrier isolates was mainly due to subtype rather than serogroup: serotype. These results are in agreement with the hypothesis that the pathogenic meningococcal population is structured in discrete subtype combinations by the action of the human immune system against the polymorphic epitopes of the outer membrane protein PorA [35]. Moreover, the proportion of ETs with more than one isolate per ET was higher among patients than carriers, as reflected by the twofold increase in the isolate/ET ratio.

PFGE confirmed all the related patient strains defined by MEE but one. In addition, PFGE identified subgroups of related PTs within the vast majority of multiple ETs, suggesting that it can resolve the microvariation within $N$. meningitidis hyper-virulent lineages circulating within a geographic area [23, 24]. Conversely, PFGE corroborated the related strains defined by MEE in only almost half of the carrier strains included in multiple ETs. Thus, by two different typing systems, $30 \%$ of carrier isolates were assigned to clonal groups including identical genotypes by MEE and identical or closely related by PFGE. This percentage was increased to $46 \%$ when carrier isolates related at a genetic distance $\leqslant 0.34$ by MEE were included.

Since recombination did not prevent the identification of the hyper-virulent clones among patients (previously recovered from other countries since the 1980s) by both typing techniques, our data provide evidence for a considerable stability of the clones associated with disease. In contrast, despite the limited number of clonal groups (by both techniques) among the carrier population, more than half of the nasopharyngeal isolates from healthy carriers showed inconsistent results by PFGE and MEE, i.e. some isolates with indistinguishable PFGE patterns were unrelated by MEE and vice versa. These data support the occurrence of frequent recombination in meningococci [36-38], making it difficult to reconstruct the network of relationships between carrier isolates and those that cause disease, as mentioned above. Therefore, phylogenetic inferences from the general meningococcal population using MEE should be interpreted cautiously.

Although virulent clonal groups arise, diversify and decay throughout the years through genetic recombination, immune selection and mutation $[15,18,24$, $39,40]$, the dynamics of meningococcal carriage remains shrouded. Our data show that non-pathogenic clusters of genetically related carrier isolates were represented by no more than $15 \%$ of strains and pathogenic clusters (i.e. clusters associated with hypervirulent lineages) by no more than $9 \%$ of carrier strains, and that transmission is infrequent in the overall population of meningococci. Moreover, we failed to detect any epidemiological connection between $63 \%$ of carriers of strains with the same ET and PFGE patterns, suggesting that these clones are commonly found among carriers, and supporting the concept of low transmission rates. Similarly, in a randomly sampled carrier population, Caugant et al. [41] were unable to identify connections between carriers included in multiple ETs and reported that some ETs were repeatedly recovered in several surveys performed in Norway [25]. Our data suggested stability not only of virulent meningococcal clones, but also of carrier clones as defined by two molecular typing methods (i.e. nearly one-third of all endemic strains recovered from symptom-free carriers in our geographically defined human population). This hints at a weakly clonal structure for the $N$. meningitidis population as a whole. However, the lack of data on the rates of recombinational exchanges makes it impossible to assess the persistence of carrier clonal groups in the population.

We have provided evidence that the use of multiple markers and, especially, the analysis of overall populations of strains, provide a better understanding of the dynamics of meningococcal carriage and the association between such carriage and sporadic meningococcal disease. Accurate strain characterization will inform future studies on both meningococcal population structure and bacterial virulence, and will be augmented by the recently developed technique of MLST and the publication of the complete genome sequence of $N$. meningitidis strain MC58. 


\section{ACKNOWLEDGEMENTS}

This research was supported by grants $94 / 1442$ and 95/0417 from the Fondo de Investigaciones Sanitarias, Spain.

\section{REFERENCES}

1. Cartwright KAV, Stuart JM, Jones DM, Noah ND. The Stonehouse survey: nasopharyngeal carriage of meningococci and Neisseria lactamica. Epidemiol Infect 1987; 99: 591-601.

2. Fontanals D, Van Esso D, Pons I, et al. Estudio de la prevalencia de portadores de Neisseria meningitidis en la población de Cerdanyola (Barcelona). Enferm Infec Microbiol Clin 1995; 13: 398-405.

3. Maiden MCJ, Feavers IM. Populations genetics and global epidemiology of the human pathogen Neisseria meningitidis. In: Baumberg S, Young JPW, Wellington EMH, Saunders JR, eds. The population genetics of bacteria. Cambridge: Cambridge University Press, 1995: 269-93.

4. Connolly $\mathrm{M}$, Noah N. Is group $\mathrm{C}$ meningococcal disease increasing in Europe? A report of surveillance of meningococcal infection in Europe 1993-6. Epidemiol Infect 1999; 122: 41-9.

5. Tikhomirov E, Santamaria M, Esteves K. Meningococcal disease: public health burden and control. World Health Stat Q. 1997; 50: 170-7.

6. Berrón S, De La Fuente L, Martín E, Vázquez JA. Increasing incidence of meningococcal disease in Spain associated with a new variant of serogroup C. Eur J Clin Microbiol Infect Dis 1998; 17: 85-9.

7. van Deuren M, Brandtzaeg P, van der Meer JWM. Update on meningococcal disease with emphasis on pathogenesis and clinical management. Clin Microbiol Rev 2000; 13: 144-66.

8. Greenwood BM. Meningococcal infections. In: Weatherall DJ et al., eds. Oxford textbook of medicine. Oxford: Oxford University Press, 1996: 533-44.

9. Verdú ME, Coll P, Fontanals D, et al. Comparison of conventional ribotyping and PCR-RFLP ribotyping for the analysis of endemic strains of Neisseria meningitidis isolated in a local community over 7 years. FEMS Microbiol Lett 1999; 179: 247-53.

10. Woods TC, Helsel LO, Swaminathan B, et al. Characterization of Neisseria meningitidis serogroup C by multilocus enzyme electrophoresis and ribosomal DNA restriction profiles (ribotyping). J Clin Microbiol 1992; 30: $132-7$.

11. Verdú ME, Coll P, Fontanals D, et al. Endemic meningococcal disease in Cerdanyola, Spain, 19871993: molecular epidemiology of the isolates of Neisseria meningitidis. Clin Microbiol Infect 1996; 2: 168-78.

12. Yakubu DE, Pennington TH. Epidemiological evaluation of Neisseria meningitidis serogroup B by pulsed- field gel electrophoresis. FEMS Immunol Med Microbiol 1995; 10: 185-9.

13. Bygraves JA, Maiden MC. Analysis of the clonal relationships between strains of Neisseria meningitidis by pulsed field gel electrophoresis. J Gen Microbiol 1992; 138: 523-31.

14. Selander RK, Caugant DA, Ochman H, Musser JM, Gilmour MN, Whittan TS. Methods of multilocus enzyme electrophoresis for bacterial population genetics and systematics. Appl Environ Microbiol 1986; 51: 873-84.

15. Caugant DA, Frøholm LO, Bøvre K, et al. Intercontinental spread of a genetically distinctive complex of clones of Neisseria meningitidis causing epidemic disease. Proc Natl Acad Sci USA 1986; 83: 4927-31.

16. Wang JF, Caugant DA, Morelli G, Koumaré B, Achtman M. Antigenic and epidemiologic properties of the ET-37 complex of Neisseria meningitidis. J Infect Dis 1993 ; 167: 1320-9.

17. Wang JF, Caugant DA, Li X, et al. Clonal and antigenic analysis of serogroup A Neisseria meningitidis with particular reference to epidemiological features of epidemic meningitis in the People's Republic of China. Infect Immun 1992; 60: 5267-82.

18. Maiden MCJ, Bygraves JA, Feil E, et al. Multilocus sequence typing: a portable approach to the identification of clones within populations of pathogenic microorganisms. Proc Natl Acad Sci USA 1998; 95 : 3140-5.

19. Feavers IM, Gray SJ, Urwin R, et al. Multilocus sequence typing and antigen gene sequencing in the investigation of a meningococcal disease outbreak. J Clin Microbiol 1999; 37: 3883-7.

20. Woods JP, Kersulyte D, Tolan RW Jr, Berg CM, Berg DE. Use of arbitrarily primed polymerase chain reaction analysis to type disease and carrier strains of Neisseria meningitidis isolated during a university outbreak. J Infect Dis 1994; 169: 1384-9.

21. Swaminathan B, Matar GM, Reeves MW, et al. Molecular subtyping of Neisseria meningitidis serogroup B: comparison of five methods. J Clin Microbiol 1996; 34: 1468-73.

22. Yakubu DE, Abadi FJR, Pennington TH. Molecular typing methods for Neisseria meningitidis. J Med Microbiol 1999; 48: 1055-64.

23. Vogel U, Morelli G, Zurth K, et al. Necessity of molecular techniques to distinguish between Neisseria meningitidis strains isolated from patients with meningococcal disease and from their healthy contacts. J Clin Microbiol 1998; 36: 2465-70.

24. Raymond NJ, Reeves M, Ajello G, et al. Molecular epidemiology of sporadic (endemic) serogroup C meningococcal disease. J Infect Dis 1997; 176: 1277-84.

25. Caugant DA, Kristiansen BE, Frøholm LO, Bøvre K, Selander RK. Clonal diversity of Neisseria meningitidis from a population of asymptomatic carriers. Infect Immun 1988; 56: 2060-8.

26. Caugant DA, Bøvre K, Gaustad P, et al. Multilocus genotypes determined by enzyme electrophoresis of 
Neisseria meningitidis isolated from patients with systemic disease and from healthy carriers. J Gen Microbiol 1986; 132: 641-52.

27. Smith JM, Smith NH, O'Rourke M, Spratt BG. How clonal are bacteria? Proc Natl Acad Sci USA 1993; 90 : 4384-8.

28. Abdillahi H, Poolman JT. Whole-cell ELISA for typing Neisseria meningitidis with monoclonal antibodies. FEMS Microbiol Lett 1987; 48: 367-71.

29. Berrón S, Vázquez JA. Increase in moderate penicillin resistance and serogroup $\mathrm{C}$ in meningococcal strains isolated in Spain. Is there any relationship? Clin Infect Dis 1994; 18: 161-5.

30. Sneath PHA, Sokal RR. Numerical taxonomy. San Francisco: WH Freeman, 1973: 230-4.

31. Tenover FC, Arbeit RD, Goering RV, et al. Interpreting chromosomal DNA restriction patterns produced by pulsed-field gel electrophoresis: criteria for bacterial strain typing. J Clin Microbiol 1995; 33: 2233-9.

32. Caugant DA, Mocca LF, Frasch CE, Frøholm LO, Zollinger WD, Selander RK. Genetic structure of Neisseria meningitidis populations in relation to serogroup, serotype, and outer membrane protein pattern. J Bacteriol 1987; 169: 2781-92.

33. Andersen J, Berthelsen L, Bech Jensen B, Lind I. Dynamics of the meningococcal carrier state and characteristics of the carrier strains: a longitudinal study within three cohorts of military recruits. Epidemiol Infect 1998; 121: 85-94.

34. Bevanger L, Bergh K, Gisnas G, Caugant DA, Frøholm LO. Identification of nasopharyngeal carriage of an outbreak strain of Neisseria meningitidis by pulsed-field gel electrophoresis versus phenotypic methods. J Med Microbiol 1998; 47: 993-8.

35. Gupta S, Maiden MCJ, Feavers IM, Nee S, May RM, Anderson RM. The maintenance of strain structure in populations of recombining infectious agents. Nature Med 1996; 2: 437-42.

36. Holmes EC, Urwin R, Maiden MC. The influence of recombination of the population structure and evolution of the human pathogen Neisseria meningitidis. Mol Biol Evol 1999; 16: 741-9.

37. Feil EJ, Maiden MCJ, Achtman M, Spratt BG. The relative contributions of recombination and mutation to the divergence of clones of Neisseria meningitidis. Mol Biol Evol 1999; 16: 1496-502.

38. Feil EJ, Enright MC, Spratt BG. Estimating the relative contributions of mutation and recombination to clonal diversification: a comparison between Neisseria meningitidis and Streptococcus pneumoniae. Res Microbiol 2000; 151: 465-9.

39. Achtman M. Clonal spread of serogroup A meningococci: a paradigm for the analysis of microevolution in bacteria. Mol Microbiol 1994; 11: 15-22.

40. Swartley JS, Marfin AA, Edupuganti S, et al. Capsule switching of Neisseria meningitidis. Proc Natl Acad Sci USA 1997; 94: 271-6.

41. Caugant DA, Hoiby EA, Magnus $P$, et al. Asymptomatic carriage of Neisseria meningitidis in a randomly sampled population. J Clin Microbiol 1994; 32: $323-30$. 